Z. Phys. Chem. 216 (2002) 235-247

(C) by Oldenbourg Wissenschaftsverlag, München

\title{
Towards a Calculus of Biological Networks
}

\author{
By H. S. Mortveit* and C. M. Reidys \\ Los Alamos National Laboratory, D-2, 87545 New Mexico, USA \\ Dedicated to Prof. Dr. Peter Schuster \\ on the occasion of his 60th birthday
}

(Received July 2, 2001; accepted July 20, 2001)

\section{Sequential Dynamical Systems / Graph Morphisms / Phase Space Embedding}

In this paper we present a new framework for studying the dynamics of biological networks. A specific class of dynamical systems, Sequential Dynamical Systems (SDS), is introduced. These systems allow one to investigate the interplay between structural properties of the network and its phase space. We will show in detail how to find a reduced system that captures key features of a given system. This reduction is based on a special graph-theoretic relation between the two networks. We will study the reduction of SDS over $n$-cubes in detail and we will present several examples.

\section{Introduction}

Biological networks, like metabolic or regulatory networks, as well as networks in general, can be considered as undirected or directed graphs in which the vertices have states that depend on the states of their corresponding adjacencies. One typically has some information about how state transitions of the respective vertices occur, but it is extremely difficult to analyze the global dynamics of the networks as interactions among the vertices occur. Additionally, it appears to be a generic feature of biological networks that these interactions take place sequentially.

Accordingly, a mathematical framework designed for the analysis of the dynamics of biological networks should explicitly take into account: scheduling, the properties of the vertices as state transition functions, and the interconnection scheme, i.e.the graph itself.

In the following we will introduce a new class of dynamical systems called sequential dynamical systems, or SDS for short [5, 10]. SDS are basically com-

* Corresponding author. E-mail: henning@lanl.gov 
prised of $i$ ) state transition rules, $i i)$ an undirected graph where each vertex has a state, and iii) a permutation of the vertices in the graph giving the update order. Thus an SDS is a dynamical system of the form $\phi: \mathbb{F}_{2}^{n} \rightarrow \mathbb{F}_{2}^{n}$, where $\mathbb{F}_{2}=\{0,1\}$ is the field with two elements.

The question of obtaining information about a network thus becomes a question of understanding the phase space structure of the SDS $\phi$. For some results on, e.g., reversibility/invertibility and fixed points we refer to $[2,3,5,10,11]$.

The phase space of an SDS will usually consist of more than one attractor. Thus a time series will only visit certain parts of phase space. Likewise for networks: there will typically be valid states or regimes that are never realized under the time evolution. It seems fair to believe that constructing a "reduced" network that produces the same dynamics as the original network in the essential regimes and "throws away" the non-essential regimes should allow for more insight and has an obvious computational advantage.

In the following we will show how to reduce the phase space of certain SDS $\phi$ over a graph $Y$. We will do so by considering an SDS $\psi$ over a smaller graph $Z$ for which there is a covering map (locally bijective graph morphism) $p: Y \rightarrow Z$. In this construction the state transition functions of corresponding $Y$ and $Z$ vertices are identical, and the dynamics of the SDS $\psi$ represents the essential parts of the dynamics of $\phi$. In fact, there can be several covering maps $p_{i}: Y \rightarrow Z_{i}$, each of which gives rise to a reduced system. This may be viewed as factorization of the SDS over $Y$ into factors which are SDS over $Z_{i}$.

Let $Y$ be a labeled graph with vertex-set $\mathrm{v}[Y]=\mathbb{N}_{n}=\{1,2,3, \ldots, n\}$, which we write as $Y<K_{n}$, where $K_{n}$ is the complete graph on $n$ vertices. The edge-set of $Y$ is denoted by e[Y]. Let $S_{1, Y}(i)$ be the set of $Y$-vertices that are adjacent to vertex $i$, let $\delta_{i}=\left|S_{1, Y}(i)\right|$ and let $d=\max _{i \in \mathbb{N}_{n}} \delta_{i}$. The increasing sequence of elements of $S_{1, Y}(i)$ preceded by $i$ is denoted by

$$
\tilde{B}_{1, Y}(i)=\left(i, j_{1}, \ldots, j_{\delta_{i}}\right) .
$$

To each vertex $i$ we associate a state $x_{i} \in \mathbb{F}_{2}$, and we write $x=\left(x_{1}, x_{2}, \ldots, x_{n}\right)$ for the system state. For each $k=1, \ldots, d+1$ we have a symmetric function $f: \mathbb{F}_{2}^{k} \rightarrow \mathbb{F}_{2}$, and for each vertex $i$ we introduce a map

$$
\operatorname{proj}_{Y}[i]: \mathbb{F}_{2}^{n} \rightarrow \mathbb{F}_{2}^{\delta_{i}+1}, \quad\left(x_{1}, \ldots, x_{n}\right) \mapsto\left(x_{i}, x_{j_{1}}, \ldots, x_{j_{\delta_{i}}}\right) .
$$

The map projects from the full $n$-tuple $x$ down to the states vertex $i$ needs for updating its state. For each $i \in \mathbb{N}_{n}$ there is a $Y$-local map $F_{i, Y}: \mathbb{F}_{2}^{n} \rightarrow \mathbb{F}_{2}^{n}$ given by

$$
\begin{aligned}
y_{i} & =f_{\delta_{i}+1} \circ \operatorname{proj}_{Y}[i], \\
F_{i, Y}(x) & =\left(x_{1}, \ldots, x_{i-1}, y_{i}(x), x_{i+1}, \ldots, x_{n}\right) .
\end{aligned}
$$

The function $F_{i, Y}$ updates the state of vertex $i$ and leaves all other states fixed. We refer to the sequence $\left(F_{i, Y}\right)_{i}$ as $F_{Y}$. Note that for each graph $Y<K_{n}$ a se- 
quence $\left(f_{k}\right)_{1 \leq k \leq n}$ induces a sequence $F_{Y}$, i.e.we have a map $\left\{Y<K_{n}\right\} \rightarrow\left\{F_{Y}\right\}$. We define the map $\left[F_{Y},\right]: S_{n} \rightarrow \operatorname{Map}\left(\mathbb{F}_{2}^{n}, \mathbb{F}_{2}^{n}\right)$ by

$$
\left[F_{Y}, \pi\right]=\prod_{i=1}^{n} F_{\pi(i), Y},
$$

where product denotes ordinary function composition.

Definition 1 (Sequential dynamical system). Let $Y<K_{n}$, let $\left(f_{k}\right)_{k}$ with $1 \leq k \leq d(Y)+1$ be a sequence of symmetric functions, and let $\pi \in S_{n}$ The sequential dynamical system (SDS) over $Y$ induced by $\left(f_{k}\right)_{k}$ with respect to the ordering $\pi$ is $\left[F_{Y}, \pi\right]$.

We call an SDS homogeneous if it is induced by a sequence of local symmetric functions of the form $\left(f_{k}\right)_{k}=\left(B_{k}\right)_{k}$ where $B$ is a Boolean function like, e.g., parity which returns the sum of its arguments modulo 2 .

Example 1. Let $Y=\mathrm{Circ}_{4}$ as shown in Fig. 1. With the parity function, i.e.par ${ }_{3}$ : $\mathbb{F}_{2}^{3} \rightarrow \mathbb{F}_{2}$ defined by $\operatorname{par}_{3}\left(x_{1}, x_{2}, x_{3}\right)=\sum_{i} x_{i} \bmod 2$, update order $(1,2,3,4)$ and initial state $(1,1,0,0)$ we get

$$
\begin{aligned}
\operatorname{Par}_{1}(1,1,0,0) & =(0,1,0,0), \\
\operatorname{Par}_{2} \circ \operatorname{Par}_{1}(1,1,0,0) & =(0,1,0,0), \\
\operatorname{Par}_{3} \circ \operatorname{Par}_{2} \circ \operatorname{Par}_{1}(1,1,0,0) & =(0,1,1,0), \\
\operatorname{Par}_{4} \circ \operatorname{Par}_{3} \circ \operatorname{Par}_{2} \circ \operatorname{Par}_{1}(1,1,0,0) & =(0,1,1,1) .
\end{aligned}
$$

and thus $\left[\operatorname{Par}_{\mathrm{Circ}_{4}},(1,2,3,4)\right](1,1,0,0)=(0,1,1,1)$.

Since phase space for an SDS is finite we may identify it with a finite unicyclic digraph.

Definition 2. The digraph $\Gamma\left[F_{Y}, \pi\right]$ associated to the $\operatorname{SDS}\left[F_{Y}, \pi\right]$ is the directed graph having vertex-set $\mathbb{F}_{2}^{n}$ and directed edges $\left\{\left(x,\left[F_{Y}, \pi\right](x)\right) \mid x \in \mathbb{F}_{2}^{n}\right\}$. A subgroup $H<Q_{2}^{n}$ will under certain conditions induce a locally bijective map $\phi: Q_{2}^{n} \rightarrow H \backslash Q_{2}^{n}$. The graph $H \backslash Q_{2}^{n}$ has vertex set $Q_{2}^{n} / H$ (factor space) and two vertices $\bar{u}, \bar{v}$ are adjacent iff there are elements $u \in \bar{u}$ and $v \in \bar{v}$ such that $\{u, v\} \in \mathrm{e}\left[Q_{2}^{n}\right]$.

This paper is organized as follows. First we introduce the concept of graph coverings and locally bijective graph morphisms. Assuming we have a graph

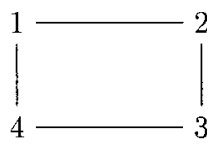

Fig. 1. The circle graph on 4 vertices, $\mathrm{Circ}_{4}$. 
covering $\phi: Y \rightarrow Z$, we show how the phase space of an SDS over $Z$ is embedded into the phase space of an SDS over $Y$ where the same local functions are used. Finally, we develop a criterion based on local bijectivity and subgroup structure of the $n$-cube $Q_{2}^{n}$ for the existence of covering maps of the form $\phi: Q_{2}^{n} \rightarrow Z=H \backslash Q_{2}^{n}, H<Q_{2}^{n}$.

\section{Factorization}

Recall that a morphism between graphs $Y$ and $Y^{\prime}$ is a pair $\phi=\left(\phi_{1}, \phi_{2}\right)$ with $\phi_{1}$ : $\mathrm{v}[Y] \rightarrow \mathrm{v}\left[Y^{\prime}\right]$ and $\phi: \mathrm{e}[Y] \rightarrow \mathrm{e}\left[Y^{\prime}\right]$ such that

$$
\forall e=\{i, j\} \in \mathrm{e}[Y]: \quad \phi_{2}(e)=\left\{\phi_{1}(i), \phi_{1}(j)\right\} .
$$

Thus, adjacent vertices in $Y$ are mapped to $i$ ) adjacent vertices in $Y^{\prime}$ or $i i$ ) to the same vertex in $Y^{\prime}$. A morphism of directed graphs also preserves the direction of edges.

A graph morphism $\phi: Y \rightarrow Y^{\prime}$ is locally bijective (surjective) if

$$
\forall i \in \mathrm{v}[Y]:\left.\phi\right|_{B_{1, Y}(i)}: B_{1, Y}(i) \rightarrow B_{1, Y^{\prime}}(\phi(i))
$$

is bijective (surjective). Note that a locally bijective graph morphism does not have to be bijective as the following example shows.

Example 2. As an example of a locally bijective graph morphism we have $\phi$ : $Q_{2}^{3} \rightarrow K_{4}$, see Fig. 2. The map $\phi_{1}$ is defined by $\phi_{1}(\{0,7\})=\{1\}, \phi_{1}(\{1,6\})=$ $\{2\}, \phi_{1}(\{2,5\})=\{3\}, \phi_{1}(\{3,4\})=\{4\}$, and $\phi_{2}$ is the induced edge-map. The resulting graph morphism is clearly bijective.

As a trivial example of locally surjective graph morphism we have $\psi: \mathrm{Star}_{4} \rightarrow$ $\mathrm{Star}_{3}$, as shown in Fig. 3. Here $\phi_{1}$ is defined by $\phi_{1}(0)=0, \phi_{1}(1)=1, \phi_{1}(2)=2$, $\phi_{1}(3)=2$.

Definition 3. Let $\left[F_{Z}, \sigma\right]$ and $\left[F_{Y}, \pi\right]$ be two SDS. An SDS-morphism between $\left[F_{Z}, \sigma\right]$ and $\left[F_{Y}, \pi\right]$ is a pair $(\phi, \Phi)$ where $\phi: Y \rightarrow Z$ is a graph morphism and where $\Phi: \Gamma\left[F_{Z}, \sigma\right] \rightarrow \Gamma\left[F_{Y}, \pi\right]$ is digraph morphism.
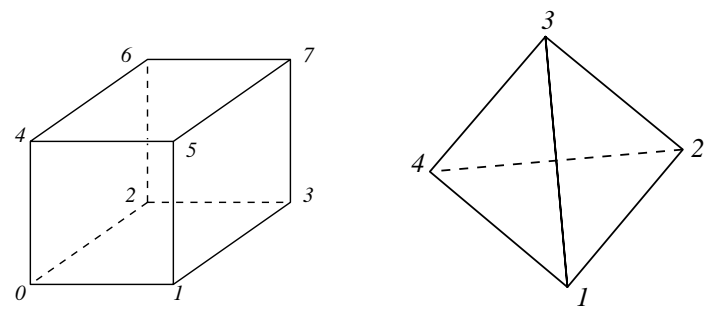

Fig. 2. The graphs $Q_{2}^{3}$ and $K_{4}$. 

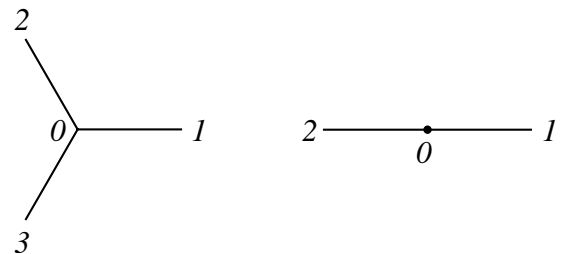

Fig. 3. The graphs $\mathrm{Star}_{3}$ and $\mathrm{Star}_{2}$.

Given a graph morphism $\phi: Y \rightarrow Z$ we want to relate the dynamics of SDS over the two graphs $Y$ and $Z$. The local functions will be the same for the two graphs unless otherwise stated. To begin, we relate update schedules for $Y$ and $Z$ via $\phi$. Assume $|\mathrm{v}[Y]|=n$ and $|\mathrm{v}[Z]|=m$ and let $\phi^{-1}(i)=\left\{i_{1}, \ldots, i_{l_{i}}\right\}$ where $i_{1}<\ldots i_{l_{i}}$ for $1 \leq i \leq m$. Define the map $\eta_{\phi}: S_{m} \rightarrow S_{n}$ by

$$
\eta_{\phi}\left(\pi=\left(\pi_{1}, \pi_{2}, \ldots, \pi_{m}\right)\right)=\left(\pi_{11}, \ldots, \pi_{1 l_{\pi_{1}}}, \ldots, \pi_{m 1}, \ldots, \pi_{1 l_{\pi_{m}}}\right) .
$$

For instance, in the example with $\phi: Q_{2}^{3} \rightarrow K_{4}$, we have $\eta_{\phi}(4,3,2,1)=$ $(3,4,2,5,1,6,0,7)$.

Similarly, we define the map $\tau: \mathbb{F}_{2}^{m} \rightarrow \mathbb{F}_{2}^{n}$ by

$$
(\tau(x))_{k}=x_{\phi(k)} .
$$

The dynamics of SDS over $Y$ and $Z$ can now be related in the following way [12]:

Theorem 1. Let $Y$ and $Z$ be loop-free connected graphs, let $\phi: Y \rightarrow Z$ be a locally bijective graph morphism, and let $\left(f_{i}\right)_{i}$ be a fixed sequence of Boolean symmetric functions. Then the map $\tau$ induces a natural embedding

$$
T: \Gamma\left[F_{Z}, \pi\right] \hookrightarrow \Gamma\left[F_{Y}, \eta_{\phi}(\pi)\right] .
$$

Example 3. To illustrate the implications of Theorem 1 we show how to relate the phase space of $\left[\boldsymbol{M i n}_{K_{4}}, \mathrm{id}_{4}\right]$ to that of $\left[\boldsymbol{M i n}_{Q_{2}^{3}},(0,7,1,6,2,5,3,4)\right]$. From the example above we have the bijective graph morphism $\phi: Q_{2}^{3} \longrightarrow K_{4}$. Next note that $\eta_{\phi}\left(\mathrm{id}_{4}\right)=(0,7,1,6,2,5,3,4)$. From, e.g., [1] we know that $\left[\operatorname{Min}_{K_{4}}, \mathrm{id}_{4}\right]$ has exactly two 5-cycles and no fixed points. The two 5-cycles are shown in the top row of Fig. 4. For convenience we use the map

$$
\xi_{i}: \mathbb{F}_{2}^{i} \longrightarrow \mathbb{N}, \quad \xi_{i}\left(x_{1}, \ldots, x_{i}\right)=\sum_{j=0}^{i} x_{j} \cdot 2^{j-1}
$$

to encode states (binary tuples), and we have, e.g., $(1,1,0,1) \mapsto 1+2+8=11$. It is straightforward to see that the phase space of [ $\left[\mathbf{M i n}_{K_{4}}, \mathrm{id}_{4}\right]$ is indeed embedded in the phase space of $\left[\operatorname{Min}_{Q_{2}^{3}},(0,7,1,6,2,5,3,4)\right]$. 


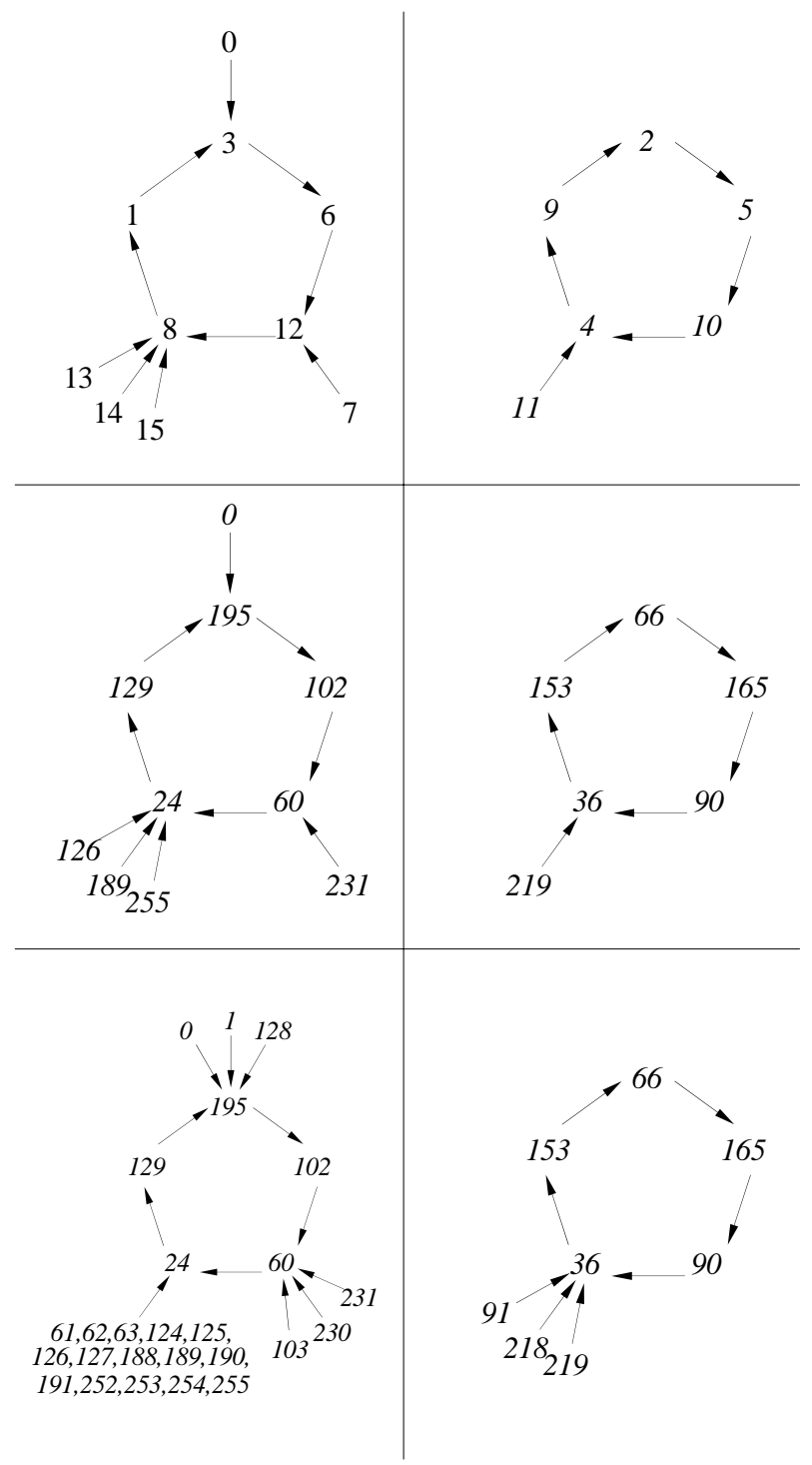

Fig. 4. The top row shows the two five-cycles in [Min ${ }_{K_{4}}$, id]. The second row shows the images of the top cycles under $\tau_{\phi}$, and the last row shows the corresponding periodic cycles in the digraph $\Gamma\left[\operatorname{Min}_{Q_{2}^{3}}, \eta_{\phi}\left(\mathrm{id}_{4}\right)\right]$.

We remark that $\left[\operatorname{Min}{ }_{Q_{2}^{3}}, \eta_{\phi}\left(\mathrm{id}_{4}\right)\right]$ has two fixed points in addition to the two 5-cycles shown in the last row in Fig. 4. These fixed points are related by the graph automorphism $\gamma=(07)(16)(25)(34)$, and consequently, so are their 


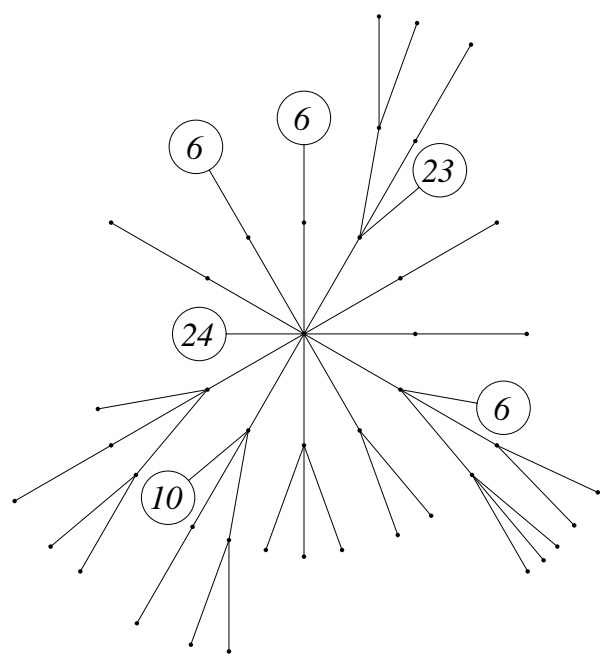

Fig. 5. The structure of the components in $\Gamma\left[\operatorname{Min}_{Q_{2}^{3}}, \eta_{\phi}\left(\mathrm{id}_{4}\right)\right]$ containing a fixed point. A single filled circle depicts a single state, while a circled number $i$ depicts that there are $i$ direct predecessors that do not have any successors themselves.

transients. Stated differently, the two components in $\Gamma\left[\operatorname{Min}_{Q_{2}^{3}}, \eta_{\phi}\left(\mathrm{id}_{4}\right)\right]$ containing the fixed points are isomorphic. Their structure is shown in Fig. 5.

In view of example 3 it is of interest to determine the locally bijective graph morphisms $\psi: Y \rightarrow Z$ for a given graph $Y$. In the particular case of a locally bijecitve graph morphism $\psi: Q_{2}^{n} \rightarrow K_{n+1}$ we have that it exists if and only if a specific number theoretic condition is fulfilled.

Proposition 1. Assume $2^{n} \equiv 0 \bmod n+1$, and let $\pi \in S_{n+1}$. Then there exists a covering $\phi: Q_{2}^{n} \rightarrow K_{n+1}$ and the SDS $\left[\operatorname{Par}_{Q_{2}^{n}}, \eta_{\phi}(\pi)\right]$ has a periodic orbit of length $n+2$.

Proof. We will establish the existence of the covering map $\phi: Q_{2}^{n} \rightarrow K_{n+1}$ under the above condition in the next section. Since a covering map is locally bijective, we can apply Theorem 1 to deduce that the phase space of $\Phi=\left[\operatorname{Par}_{K_{n}}, \pi\right]$ can be embedded into the phase space of $\Psi=\left[\operatorname{Par}_{Q_{2}^{n}}, \eta_{\phi}(\pi)\right]$. Thus we see that whatever we can deduce about the smaller system $\Phi$ applies to the larger system $\Psi$. The phase space of $\Psi$ has $2^{2^{n}}$ points while that of $\Phi$ has $2^{n+1}$ points.

One particular consequence of this is that every periodic orbit for $\Phi$ will also be a periodic orbit for $\Psi$. We will show that $\Phi$ always have a periodic orbit of length $n+2$. For simplicity we take $\pi=\mathrm{id}_{n+1}$.

By inspection $\operatorname{par}_{n}: \mathbb{F}_{2}^{n} \rightarrow \mathbb{F}_{2}^{n}$ is seen to satisfy the functional relation

$$
\psi\left(x_{1}, \ldots, x_{n-1}, \psi\left(x_{1}, \ldots, x_{n}\right)\right)=x_{n} .
$$


As a consequence of this we derive

$$
\begin{aligned}
x=\left(x_{1}, x_{2}, \ldots, x_{n}\right) & \stackrel{1}{\mapsto}\left(\operatorname{par}_{n}(x), x_{2}, x_{3}, \ldots, x_{n}\right) \\
& \stackrel{2}{\mapsto}\left(\operatorname{par}_{n}(x), \operatorname{par}_{n}\left(\operatorname{par}_{n}(x), x_{2}, \ldots, x_{n}\right), x_{3}, \ldots, x_{n}\right) \\
& =\left(\operatorname{par}_{n}(x), x_{1}, x_{3}, \ldots, x_{n}\right) \\
& \vdots \\
& \stackrel{n}{\mapsto}\left(\operatorname{par}_{n}(x), x_{1}, x_{2}, \ldots, x_{n-1}\right),
\end{aligned}
$$

where $\stackrel{i}{\mapsto}$ denotes the update of state $x_{i}$.

In light of the above we obtain the commutative diagram

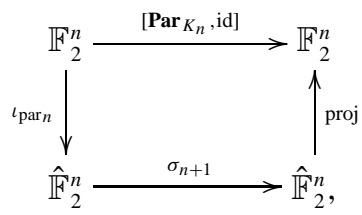

where

$$
\begin{aligned}
\operatorname{proj}\left(x_{1}, \ldots, x_{n}, x_{n+1}\right) & =\left(x_{1}, \ldots, x_{n}\right), \\
\iota_{\operatorname{par}_{n}}\left(x_{1}, \ldots, x_{n}\right) & =\left(x_{1}, \ldots, x_{n}, \operatorname{par}_{n}\left(x_{1}, \ldots, x_{n}\right)\right), \\
\sigma_{n+1}\left(x_{1}, x_{2}, \ldots, x_{n+1}\right) & =\left(x_{n+1}, x_{1}, \ldots, x_{n}\right),
\end{aligned}
$$

and $\hat{\mathbb{F}}_{2}^{n}=\left\{x \in \mathbb{F}_{2}^{n+1} \mid x_{n+1}=\operatorname{par}_{n}\left(x_{1}, \ldots, x_{n}\right)\right\}$.

Note that proj $: \hat{\mathbb{F}}_{2}^{n} \rightarrow \mathbb{F}_{2}^{n}$ and $\iota_{\mathrm{par}_{n}}: \mathbb{F}_{2}^{n} \rightarrow \hat{\mathbb{F}}_{2}^{n}$ are inverses. Similarly we obtain $\left[\operatorname{Par}_{K_{n}}, \mathrm{id}\right]^{(2)}(x)=\left(x_{n}, \operatorname{par}_{n}(x), x_{1}, x_{2}, \ldots, x_{n-2}\right)$ and in general $\left[\operatorname{Par}_{K_{n}}, \mathrm{id}\right]^{(k)}=\operatorname{proj} \circ \sigma_{n+1}^{k} \circ \iota_{\operatorname{par}_{n}}$ showing that the order of an orbit of [Par $K_{n}$, id] is a divisor of $n+1$.

Along the same lines we can show that $\left[\mathbf{P a r}_{K_{n}}, \pi\right]$ and $\left[\mathbf{P a r}_{K_{n}}\right.$, id $]$ are topologically conjugated systems. We omit the details.

Thus we have that the length of every orbit of $\left[\operatorname{Par}_{K_{n+1}}, \pi\right]$ is a divisor of $n+2$. However, it is easy to see that the orbit containing the state $(1,0,0, \ldots, 0)$ always have length $n+2$. To be explicit we have for $n=7$

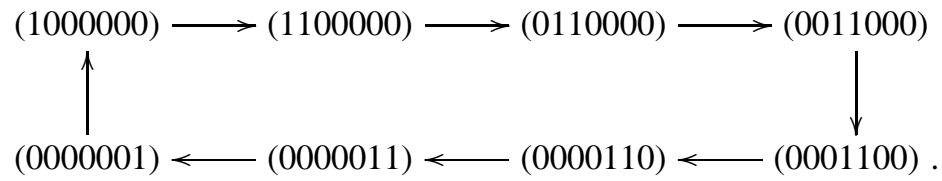

Thus we deduce from Theorem 1 that $\left[\operatorname{Par}_{Q_{2}^{n}}, \eta_{\phi}(\pi)\right]$ has a periodic orbit of length $n+2$, and we are done. 


\section{Covering maps over the $n$-cube}

Proposition 1 turns out to be a special case of a more general theory for constructing covering maps over $n$-cubes. The theory for $n$-cubes, which may also extend to, e.g., certain Cayley graphs, is facilitated by the fact that $\mathbb{F}_{2}^{n}$ can be viewed both as a group and as a vector space.

In this section we will show how the existence of subgroups $H<\mathbb{F}_{2}^{n}$ with certain properties can be used to obtain covering maps. In fact, this leads to a cascade of sequences of covering maps.

The following two results constitute the core of this theory [12]:

Lemma 1. For any subgroup $H^{\prime}<\mathbb{F}_{2}^{n}$ with $\left[\mathbb{F}_{2}^{n}: H^{\prime}\right] \geq n+1$ there exists an isomorphic subgroup $H \cong H^{\prime}$ that has the property $H(x) \cap H(y)=\varnothing$ for $x \neq$ $y ; x, y \in\left\{0, e_{1}, \ldots, e_{n}\right\}$.

The following proposition shows how the subgroups of Lemma 1 induce covering maps in a natural way:

\section{Proposition 2.}

For each subgroup $H<\mathbb{F}_{2}^{n}$ with the property $H(x) \cap H(y)=\varnothing$ for $x \neq y$; $x, y \in\left\{0, e_{1}, \ldots, e_{n}\right\}$ the graph $H \backslash \mathcal{Q}_{2}^{n}$ is connected, undirected and loop-free and the natural projection

$$
\pi_{H}: Q_{2}^{n} \longrightarrow H \backslash Q_{2}^{n}, \quad v \mapsto H(i)
$$

is a covering map.

Proof. We have to show that the $\pi_{H}$-induced restriction mapping:

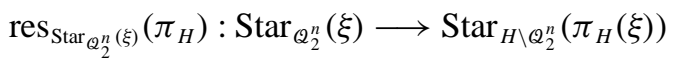

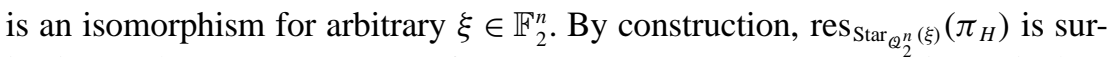
jective and $H(x) \cap H(y)=\varnothing$ for $x \neq y ; x, y \in\left\{0, e_{1}, \ldots, e_{n}\right\}^{2}$ is equivalent to $H(x+\xi) \cap H(y+\xi)=\varnothing$ for $x \neq y ; x, y \in\left\{0, e_{1}, \ldots, e_{n}\right\}$ for any $\xi \in \mathbb{F}_{2}^{n}$.

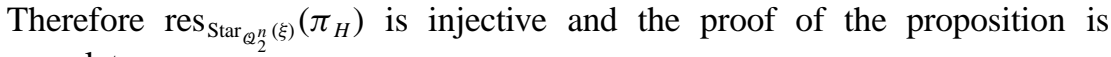
complete.

Corollary 1. Let $n$ be a natural number. Then we have $2^{n} \equiv 0 \bmod n+1$ if and only if there exists a subgroup $G<\mathbb{F}_{2}^{n}$ with the property $\mathbb{F}_{2}^{n}=G(0) \cup$ $\bigcup_{i=1}^{n} G\left(e_{i}\right)$.

Proof. Suppose we have $2^{n} \equiv 0 \bmod n+1$. Since $\mathbb{F}_{2}^{n}$ is a $p$-group there exists a subgroup in its decomposition series $H<\mathbb{F}_{2}^{n}$ with the property $\left[\mathbb{F}_{2}^{n}\right.$ : $H]=n+1$. According to Lemma 1 , there exists some set of $H$-representatives $\left\{\varphi_{1}, \ldots, \varphi_{n}\right\}$ that forms a basis of $\mathbb{F}_{2}^{n}$. Let $f$ be the $\mathbb{F}_{2}$-homomorphism defined by $f\left(\varphi_{i}\right)=e_{i}$, for $i=1, \ldots, n$. Clearly, $G=f(H)$ has the property $\mathbb{F}_{2}^{n}=G(0) \cup \bigcup_{i=1}^{n} G\left(e_{i}\right)$, whence the corollary. 
Corollary 2. There exists a locally bijective graph morphism

$$
\varphi: Q_{2}^{n} \longrightarrow K_{n+1}
$$

if and only if $2^{n} \equiv 0 \bmod n+1$ holds.

The following lemma establishes a group action of $\operatorname{Aut}_{0}\left(Q_{2}^{n}\right)=\{\sigma \in$ $\left.\operatorname{Aut}\left(Q_{2}^{n}\right) \mid \sigma(0)=0\right\}$ on graphs $H \backslash Q_{2}^{n}$.

Lemma 2. Let $H<\mathbb{F}_{2}^{n}$ be a subgroup and let $\eta \in \operatorname{Aut}_{0}\left(Q_{2}^{n}\right)$. Then we have

$$
H \backslash Q_{2}^{n} \cong \eta H \backslash Q_{2}^{n} .
$$

\subsection{Computation of covering maps}

For small $n$ we can easily compute the various subgroups of $Q_{2}^{n}$ [4]. It turns out that for fixed $n \leq 7$ the orbits of the subgroups under $\operatorname{Aut}_{0}\left(Q_{2}^{n}\right)$ (the isometric orbits) yield non-isomorphic reduced graphs. Moreover, for $n=4$ the only covering map images of $Q_{2}^{n}$ are the ones that can be obtained as $H \backslash Q_{2}^{n}$. One may speculate if this is true in general. That is, do the isometric orbits yield nonisomorphic covering images and can all covering images be obtained as $H \backslash Q_{2}^{n}$ for some subgroup $H$.

Table 1. Subgroups of the $n$-cube: The table gives the number of isomorphic-nonisometric orbits of subgroups $H<Q_{2}^{n}$ of the given sizes.

\begin{tabular}{rcccc}
\hline$n$ & $\#($ size 2$)$ & $\#($ size 4$)$ & $\#($ size 8$)$ & $\#($ size 16) \\
\hline 4 & 2 & 0 & 0 & 0 \\
5 & 3 & 1 & 0 & 0 \\
6 & 4 & 4 & 1 & 0 \\
7 & 5 & 8 & 5 & 1 \\
8 & 6 & 14 & 15 & 6 \\
9 & 7 & 22 & 38 & - \\
\hline
\end{tabular}

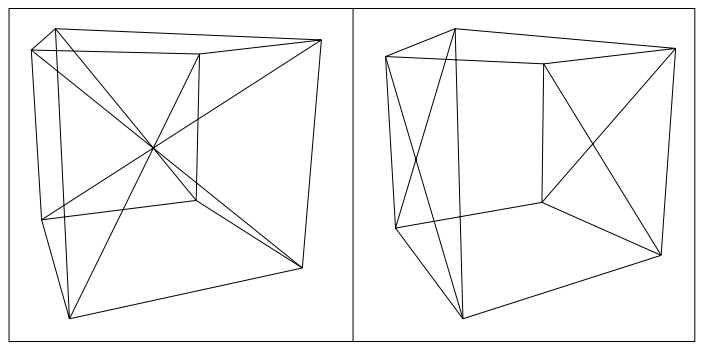

Fig. 6. The only two non-isomorphic graphs of the form $H \backslash Q_{2}^{4}$. 
Table 2. Subgroups of the $n$-cube: The table gives representatives of the isomorphic-nonisometric orbits of subgroups $H<Q_{2}^{n}$ of the given sizes that satisfy the condition in Lemma 1. Explicit computations show that the orbits give non-isomorphic reduced graphs $H \backslash Q_{2}^{n}$ for $n \leq 7$. Binary vectors have been encoded as integers.

\begin{tabular}{|c|c|c|c|c|}
\hline$n$ & Size 2 & Size 4 & Size 8 & Size 16 \\
\hline 4 & $\{0,14\},\{0,15\}$ & & & \\
\hline 5 & $\begin{array}{c}\{0,28\},\{0,30\} \\
\{0,31\}\end{array}$ & $\{0,7,25,30\}$ & & \\
\hline 6 & $\begin{array}{l}\{0,56\},\{0,60\} \\
\{0,62\},\{0,63\}\end{array}$ & $\begin{array}{c}\{0,7,25,30\}, \\
\{0,7,56,63\} \\
\{0,7,57,62\} \\
\{0,15,51,60\}\end{array}$ & $\begin{array}{c}\{0,7,25,30 \\
42,45,51,52\}\end{array}$ & \\
\hline 7 & $\begin{array}{c}\{0,112\},\{0,120\} \\
\{0,124\},\{0,126\} \\
\{0,127\}\end{array}$ & $\begin{array}{c}\{0,7,25,30\} \\
\{0,7,56,63\} \\
\{0,7,57,62\} \\
\{0,7,120,127\} \\
\{0,7,121,126\} \\
\{0,15,51,60\} \\
\{0,15,113,126\} \\
\{0,15,115,124\}\end{array}$ & $\begin{array}{c}\{0,7,25,30,42, \\
45,51,52\} \\
\{0,7,25,30,97, \\
102,120,127\} \\
\{0,7,25,30,98, \\
101,123,124\} \\
\{0,7,25,30,106, \\
109,115,116\} \\
\{0,15,51,60,85, \\
90,102,105\}\end{array}$ & $\begin{array}{c}\{0,7,25,30,42 \\
45,51,52,75 \\
76,82,85,97 \\
102,120,127\}\end{array}$ \\
\hline
\end{tabular}

In Table 1 we have summarized the number of non-isometric orbits for all subgroups of $Q_{2}^{n}$ satisfying the condition in Proposition 2 for $n \leq 9$. In Table 2 orbit representatives are given for $n \leq 7$. Some of the reduced graphs occurring in this scheme are shown in Figs. 6 and 7.

\subsection{Constructing covering graphs from chain maps}

In [6] a way of constructing graph coverings of a given graph is described. We will outline it briefly. Let $S \Gamma$ denote the set of arcs or sides of the graph $\Gamma$. Thus each edge $\{u, v\}$ gives rise to two sides, $(u, v)$ and $(v, u)$. Let $G$ be any group. A $G$-chain on $\Gamma$ is a map $\phi: S \Gamma \rightarrow G$ such that $\phi(u, v)=(\phi(v, u))^{-1}$ for all sides $(u, v)$ of $\Gamma$. The covering map $\hat{\Gamma}=\hat{\Gamma}(G, \phi)$ of $\Gamma$ with respect to a given $G$-chain $\phi$ on $\Gamma$ is the graph with $\mathrm{v}[\hat{\Gamma}]=G \times \mathrm{v}[\Gamma]$ and where vertices $\left(g_{1}, v_{1}\right)$ and $\left(g_{2}, v_{2}\right)$ are joined by an edge iff $\left(v_{1}, v_{2}\right) \in S \Gamma$ and $g_{2}=g_{1} \phi\left(v_{1}, v_{2}\right) . \hat{\Gamma}$ is clearly well-defined.

Note that the 3 -cube is isomorphic to the covering graph $\hat{K}_{4}\left(\mathbb{F}_{2}, \phi\right)$ of $K_{4}$ where $\phi$ is the $\mathbb{F}_{2}$-chain assigning 1 to each side of $K_{4}$. 


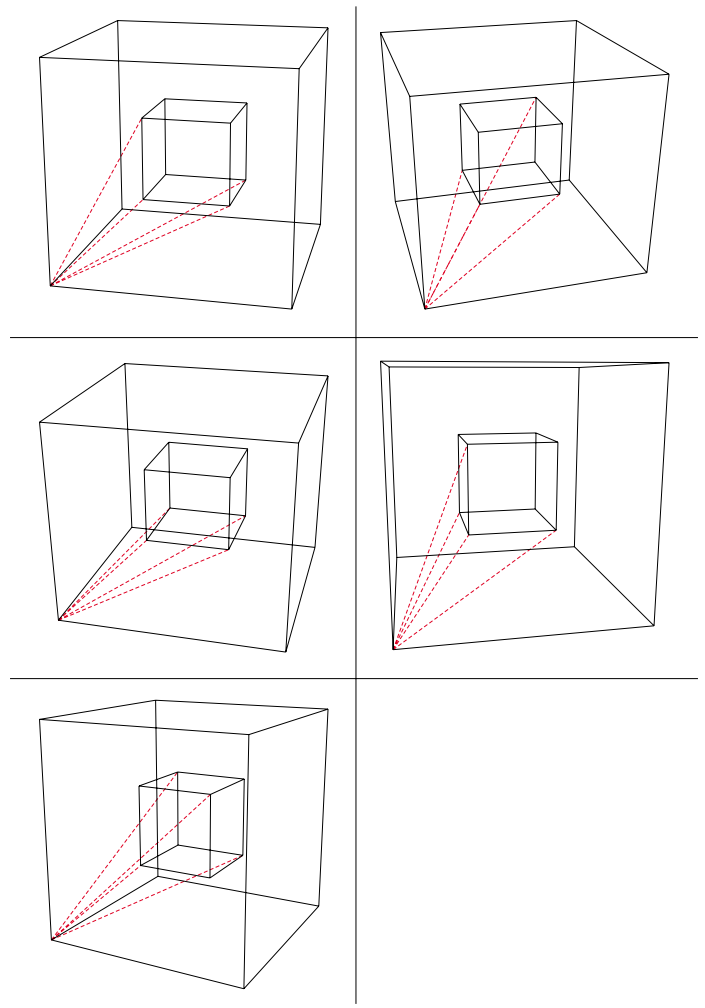

Fig. 7. All non-isomorphic graphs of the form $H \backslash Q_{2}^{7}$ on 16 vertices. In each case there are two interlocked cubes. The dashed edges show how the two cubes are connected. The other vertices of the outer cube are similarly connected, but the edges are not shown to ease visualization. Note that there are 4 lines in figure on the top right $\star$. The two lines connecting the outer cube to the closest vertex and the most distant vertex of the inner cube coincide.

Moreover we note that the graph $G_{5}$ on the third row in Fig. 7 is isomorphic to the covering graph $\hat{K}_{8}\left(\mathbb{F}_{2}, \phi\right)$, where $\phi$ again is the $\mathbb{F}_{2}$-chain assigning 1 to each side of $K_{8}$.

Another, but quite similar, approach for constructing covering maps uses so-called "voltage graph" [7-9]. Voltage graphs and $G$-chains are strongly related.

\section{Synopsis}

In this paper we have introduced a mathematical framework suitable for the analysis of network dynamics. A new class of dynamical systems, SDS, that is an abstract model for the dynamics of networks has been discussed. 
The results on SDS presented in this paper display the interplay between the structure of the underlying networks and their corresponding dynamics. Theorem 1 allows one, for particular schedules, to obtain key information about a given system via a reduced/simpler network. We have shown for the special case of Boolean $n$-cubes how to construct these reduces systems based on the subgroup structure of $\mathbb{F}_{2}^{n}$. Networks over $n$-cubes can be reduced to networks over smaller graphs induced by symmetries of the $n$-cube itself.

\section{Acknowledgement}

We thank C. L. Barrett for stimulating discussions. Special thanks and gratitude to D. Morgeson for his continuous support. This research is supported by Laboratory Directed Research and Development under DOE contract W-7405-ENG-36 to the University of California for the operation of the Los Alamos National Laboratory.

\section{References}

1. C. L. Barrett, H. S. Mortveit and C. M. Reidys, Appl. Math. Comput. 107 (2000) 121.

2. C. L. Barrett, H. S. Mortveit and C. M. Reidys, Appl. Math. Comput. 122 (2001) 325.

3. C. L. Barrett, H. S. Mortveit and C. M. Reidys, Appl. Math. Comput. (2000) in press.

4. C. L. Barrett, H. S. Mortveit and C. M. Reidys, Appl. Math. Comput. (2001) in progress.

5. C. L. Barrett and C. M. Reidys, Appl. Math. Comput. 98 (1999) 241.

6. N. Biggs, Algebraic Graph Theory, second ed., Cambridge Mathemtical Library (1993).

7. J. L. Gross, Discrete Mathematics 9 (1974) 239.

8. J. L. Gross and T. W. Tucker, Discrete Mathematics 18 (1977) 273.

9. M. Hofmeister, Discrete Mathematics 98 (1988) 175.

10. H. S. Mortveit and C. M. Reidys, Discrete Mathematics 226 (2001) 281.

11. C. M. Reidys, Adv. Appl. Math. (2000) accepted.

12. C. M. Reidys, Adv. Appl. Math. (2001) in progress. 